\title{
Capitate and hamate fracture in a child: the value of MRI imaging
}

\author{
Miryam C. Obdeijn • Cornelis van Vliet • \\ Rick R. van Rijn
}

Received: 18 February 2009 / Accepted: 14 April 2009/Published online: 26 May 2009

(C) The Author(s) 2009. This article is published with open access at Springerlink.com

\begin{abstract}
Carpal bone fractures in children are rare, and little is known about the appropriate tools to diagnose them, particularly in toddlers. We present a 2-year-old child with a capitate and hamate fracture. Based on our experiences with this case and on a review of the literature, we discuss the value of magnetic resonance imaging in carpal trauma in children.
\end{abstract}

Keywords Capitate $\cdot$ Fracture $\cdot$ Carpal $\cdot$ Child $\cdot$ Hamate

\section{Introduction}

Carpal bone fractures in children are rare [1-5]. Wulff reported an incidence of $0.39 \%$ for scaphoid fractures Other carpal fractures are even rarer and are incidentally published in case reports only [1-6]. Most of the cases described are fractures of the scaphoid and the capitate. They often occur in combination as the scaphocapitate

M. C. Obdeijn $(\bowtie)$

Department of Plastic, Reconstructive and Handsurgery,

Academic Medical Center, University of Amsterdam,

P.O. Box 22700, 1100 DE, Amsterdam, The Netherlands

e-mail: M.C.Obdeijn@amc.uva.nl

C. van Vliet

Department of Surgery, Trauma Unit, Academic Medical Center,

University of Amsterdam,

Amsterdam, The Netherlands

R. R. van Rijn

Department of Radiology, Academic Medical Center,

University of Amsterdam,

Amsterdam, The Netherlands syndrome [7]. This syndrome was first described by Fenton in 1956 [8]. However, most of these fractures have been reported in children aged 5 years and over. We present a 2year-old girl with a capitate and hamate fracture resulting from direct witnessed trauma. The value of magnetic resonance imaging (MRI) is discussed based on literature and our experience with this patient.

\section{Case report}

A 2-year-old girl was referred to the emergency department of our hospital after a blunt trauma to her left hand. While playing, it was witnessed that a drawer toppled over and fell on her hand. Radiography showed a dislocated capitate fracture (Fig. 1). At this stage of skeletal development, only the capitate and the hamate are ossified. Therefore we had no information concerning the other carpal bones. Given the possible combination of fractures, such as the scaphocapitate syndrome, and the rarity of this fracture, a noncontrastenhanced MRI scan under sedation was performed in order to ascertain the anatomical relationships within the hand. T2STIR (TR 4,000 ms, TI $25 \mathrm{~ms}$, TI 150, slice thickness $3 \mathrm{~mm}$ ) weighted imaging showed bone marrow edema in both the capitate and the hamate (Fig. 2). A distinct fracture line was seen in the hamate. There were no signs of other carpal injuries, in particular, not of a scaphoid fracture.

As the fracture was dislocated, she underwent surgery at which open reduction and internal fixation with a Kirschner wire (K-wire) of the capitate fracture was performed. Postoperative X-ray showed good reduction and fixation of the fracture (Fig. 3). The K-wire was removed after 6 weeks. Healing was uneventful, and she retained normal hand function. 


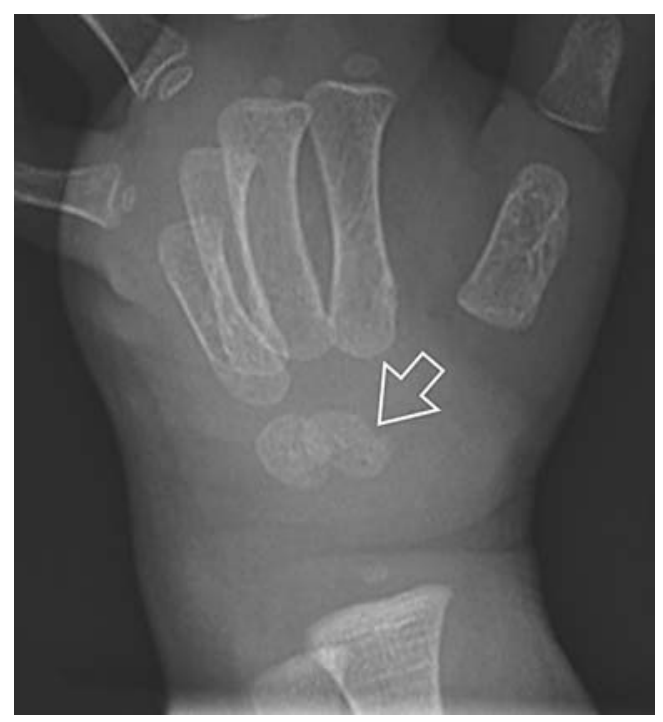

Fig. 1 Capitate fracture on conventional X-ray

\section{Discussion}

Pediatric capitate fractures are very rare, especially isolated ones [1-5]. Capitate fractures are usually seen in conjunction with scaphoid fractures the so-called scaphocapitate syndrome [6-8]. This is usually the result of a high-energy trauma. Isolated capitate fractures usually occur after a direct trauma and are easily overlooked on a plain radiograph $[9,10]$. In young children, the diagnosis is even more difficult because most of the carpal bones are not yet ossified. Fractures of the hand in young children are reported to have a strong association with non-accidental injury (NAI) $[11,12]$. These fractures may not be clinically evident and can be easily missed. Therefore, an appropriate workup with respect to NAI should be performed. This

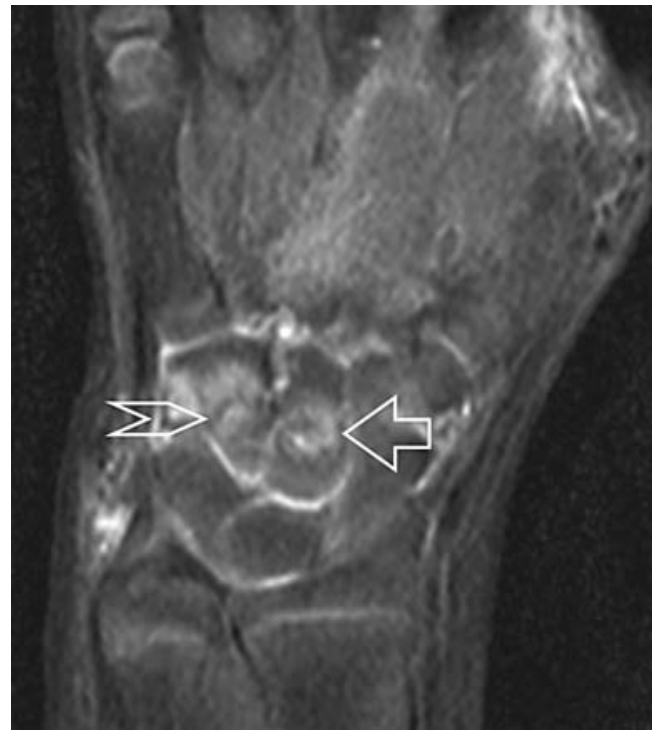

Fig. 2 Fracture on MRI image

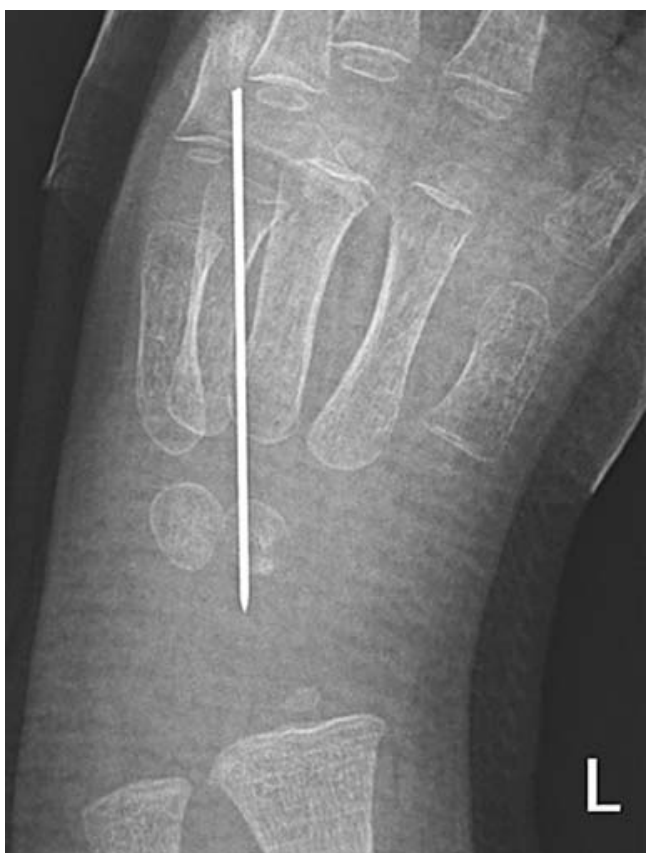

Fig. 3 Postoperative X-ray

workup consists of a thorough clinical exam by a senior pediatrician, with knowledge on the field of child abuse or by a member of the child advocacy team. In order to detect older or other clinically silent fractures, in children under the age of 2 years, a full skeletal survey according to the American College of Radiologists should be performed [13]. The radiographs should preferably be read by a pediatric radiologist. It should be stressed that the sole finding of a rare fracture in a child in itself is no proof of child abuse, but that this diagnosis should be based on a constellation of findings and can only be made after thorough discussion of all findings.

The cartilaginous components of the carpal bones can be visualized using imaging techniques such as ultrasonography [14], computed tomography, and MRI. There are only very few articles in the literature which describe the value of these imaging techniques in young children $[15,16]$. It is known from adult studies that conventional radiographs are of low negative predictive value (NPV). Dorsay et al. reported that in a meta-analysis of eight studies comprising 366 patients at high clinical suspicion, the average NPV of conventional radiographs of the hand was $74 \%$ [17]. In collaboration with our pediatric radiologist, we decided to perform MRI of the hand in order to confirm the degree of dislocation of the capitate fracture and to search for associated trauma to the other carpal bones.

In adult studies, MRI has been shown to be a sensitive technique for the detection of carpal fracture [18] and, in clinical practice, has replaced bone scintigraphy. Cook and co-workers reported on 18 skeletally immature patients. In their study, a normal MRI ruled out osseous and cartilag- 
inous lesions in $100 \%$ of patients [19]. MRI protocol should include coronal T1-weighted imaging in order to assess anatomical relationships and fracture location, and coronal STIR-weighted images, in order to assess bone marrow edema. On T1 and T2 weighted imaging the fracture line on MRI appears as a discrete linear low-signalintensity lesion. Surrounding bone marrow edema may, however, obscure subtle fractures. The different MRI sequences should be seen as complementary to each other. Breitenseher and co-workers reported on sensitivity to T1 Spin echo and STIR-weighted MRI with respect to trabecular fractures $(88 \%$ vs $88 \%)$, to cortical fractures ( $21 \%$ vs $100 \%$ ), and to edema (100\% vs $100 \%$ ) [20].

As in general preoperative MRI in a young child requires additional sedation or anesthesia in order to perform the examination, it is imperative to know whether it will provide additional information, and if it is necessary to aid in planning the optimal surgical procedure. We believe that this is indeed the case. In children in particular, the surgical exposure should be kept to a minimum in order to avoid damage to the intercarpal ligaments. By knowing which lesions to expect before the operation, surgeons can limit their exposure to the affected area and thus perform a minimally invasive open reduction and internal fixation.

In conclusion, we have shown that in this case, MRI was a useful additional diagnostic tool for the diagnosis of carpal lesions in a skeletally immature child. The MRI was able to confirm the diagnosis of a capitate fracture and to reveal the associated hamate fracture. Solely with this supplementary information, an optimal surgical procedure can be planned.

Acknowledgment The authors would like to thank Dr. S. Strackee who operated on this child.

Open Access This article is distributed under the terms of the Creative Commons Attribution Noncommercial License which permits any noncommercial use, distribution, and reproduction in any medium, provided the original author(s) and source are credited.

\section{References}

1. Wulff RN, Schmidt TL (1998) Carpal fractures in children. J Pediatr Orthop 18(4):462-465. doi:10.1097/00004694199807000-00011
2. Goddard N (2005) Carpal fractures in children. Clin Orthop Relat Res (432):73-76. doi:10.1097/01.blo.0000156003.24374.ab

3. Toh S, Miura H, Arai K, Yasumura M, Wasa M, Tsubo K (2003) Scaphoid fractures in children: problems and treatment. J Pediatr Orthop 23(2):216-221. doi:10.1097/00004694200303000-00016

4. Elhassan BT, Shin AY (2006) Scaphoid fracture in children. Hand Clin 22(1):31-41. doi:10.1016/j.hcl.2005.10.004

5. Light TR (2000) Carpal injuries in children. Hand Clin 16(4):513522

6. Kamano M, Fukushima K, Honda Y (1998) Multiple carpal bone fractures in an eleven-year-old. J Orthop Trauma 12(6):445-448. doi:10.1097/00005131-199808000-00017

7. Sawant M, Miller J (2000) Scaphocapitate syndrome in an adolescent. J Hand Surg 25(6):1096-1099

8. Fenton RL (1956) The naviculo-capitate fracture syndrome. J Bone Jt Surg 38A:681-684

9. Albertsen J, Mencke S, Christensen L, Teisen H, Hjarbak J (1993) Isolated capitate fracture diagnosed by computed tomography. Case report. Handchir Mikrochir Plast Chir 31(2):79-81. doi:10.1055/s-1999-13498

10. Calandruccio JH, Duncan SF (1999) Isolated nondisplaced capitate waist fracture diagnosed by magnetic resonance imaging. J Hand Surg [Am] 24(4):856-859. doi:10.1053/ jhsu. 1999.0856

11. Nimkin K, Spevak MR, Kleinman PK (1997) Fractures of the hands and feet in child abuse: imaging and pathologic features. Radiology 203(1):233-236

12. Offiaf A, van Rijn RR, Perez-Rossello JM, Kleinman PK (2009) Skeletal imaging of child abuse (non-accidental injury). Pediatr Radiol 39(5):461-470. doi:10.1007/s00247-009-1157-1

13. American College of Radiology ACR. Appropriateness Criteria Suspected Physical Abuse-Child. ACR, 2005. www.acr.org

14. Herneth AM, Siegmeth A, Bader TR, Ba-Ssalamah A, Lechner G, Metz VM, Grabenwoeger F (2001) Scaphoid fractures: evaluation with high-spatial-resolution US initial results. Radiology 220 (1):231-235

15. Partan G, Pamberger P, Blab E, Hruby W (2003) Common tasks and problems in paediatric trauma radiology. Eur $\mathrm{J}$ Radiol 48 (1):103-124. doi:10.1016/S0720-048X(03)00199-2

16. Riccabona M, Lindbichler F (2002) Trauma radiology in the child. Radiology 42(3):195-209. doi:10.1007/s00117-001-0709-7

17. Alzen G, Wildberger JE, Gunther RW (1995) Diagnostic imaging in the traumatized child. Radiology 35(6):373-377

18. Dorsay TA, Major NM, Helms CA (2001) Cost-effectiveness of immediate MR imaging versus traditional follow-up for revealing radiographically occult scaphoid fractures. AJR Am J Roentgenol 177:1257-1263

19. Breitseher MJ, Metz VM, Gilula LA, Gaebler C, Kukla C, Fleischmann D, Imhof H, Trattnig S (1997) Radiographically occult scaphoid fractures: value of MR imaging in detection. Radiology 203(1):245-250

20. Cook PA, Yu JS, Wiand W, Cook AJ II, Coleman CR, Cook AJ (1997) Suspected scaphoid fractures in skeletally immature patients: application of MRI. J Comput Assist Tomogr 21:511515. doi:10.1097/00004728-199707000-00001 